\title{
The sickling process in relation to clinical manifestations
}

\author{
A. J. BELLINGHAM \\ From the Department of Clinical Haematology, University College Hospital Medical School, London
}

The knowledge of the detailed structure and function of the haemoglobin molecule has led to a better understanding of the pathophysiology of the clinical syndromes associated with abnormal haemoglobins. Not least of these is the commonest haemoglobin variant seen in the UK, sickle haemoglobin (Hb-S). Sickle cell disease is characterized by painful crises and a chronic haemolytic state which stem from the haemoglobin's tendency to polymerize leading to distortion and rigidity of the red cell causing blocking of small capillaries and the red cells' premature destruction.

Haemoglobin consists of a tetramer of $2 \alpha$ and $2 \beta$ chains existing in two conformational states in equilibrium with each other: these are known as 'deoxy' and 'oxy' conformations (Perutz, 1970). This equilibrium may be moved to favour one or other of the conformations by the ability of various ligands in the environment of the haemoglobin to bind preferentially to one of the states. For example, the binding of oxygen moves the equilibrium to the 'oxy' state while hydrogen ions and a rise in temperature favours the 'deoxy' state. Inherited abnormalities of the haemoglobin molecule may also alter this equilibrium as well as causing other functional disturbances such as instability and methaemoglobin formation. By correlating the functional disturbances of abnormal haemoglobins with the site of the substituted amino acid in the haemoglobin chain it has become possible to predict the consequences of a given substitution fairly confidently (Huehns, 1973). The substitution in sickle haemoglobin $\left(\beta^{6} \mathrm{glu} \rightarrow\right.$ val $)$ is on the external surface of the molecule and would at first sight not be expected to cause any functional disturbance. Furthermore in vitro, sickle haemoglobin is stable and yet in vivo it causes chronic haemolysis. The clinical manifestations of sickle cell disease arise from the ability of one $\mathrm{HbS}$ molecule to bind to an adjacent molecule so leading to polymerization of the molecules, tactoid formation, and sickling of the red cells (Perutz, 1971). The details of the these inter-molecular binding sites have not been defined but the polymerization is conformational-dependent as it only occurs in the deoxy state which is evidenced by the standard test in vitro with metabisulphate which examines for the sickling of cells as they are deoxygenated.

Crystallographic and electron microscopic studies have shown that the tactoid formation is ordered and regular. The sickle molecules form long strands which are themselves in bundles of six having a hexagonal cross section and also having a twist rather like the spinning of a piece of thread. The twist is such that it takes a strand of $\mathbf{4 8}$ molecules to do a complete turn $\left(360^{\circ}\right)$ which means the length required for this is approximately $3000 \AA$ (Finch, Perutz, Bertles, and Dobler, 1973).

\section{Clinical Management}

From this basic information of molecular function it is possible to build up a logical approach for the management of the disease (a) by avoiding conditions in which there is predisposition for the haemoglobin to be in the 'deoxy' state, and (b) by therapeutic intervention (with drugs) either to block the site of intermolecular interaction or to shift the equilibrium in favour of the 'oxy' conformation.

There are three factors of physiological importance which tend to favour the deoxy conformation, namely, fever, acidosis, and hypoxia. The association between sickling crises and infection is well established and the results of malaria prophylaxis in reducing the incidence of crises is also well documented. In the UK fever is commonly the result of mild viral infections for which prophylaxis is generally not feasible. A young child at school is frequently subject to recurrent infections and I wonder in this age group whether the incidence of crises might be reduced if prophylactic antibiotics were used to protect from secondary infections. In the older age group such treatment has less to recommend it owing to the lower incidence of such infection, although a patient who is particularly at risk might be considered for at least a trial period of 
maintenance antibiotics. There is no doubt that reducing fever helps to maintain the health of sickle patients, and the adequate search for, and treatment of, infections is a cornerstone in treatment. In this respect the use of an antipyretic such as aspirin, which in a crisis has the benefit of also being an analgesic, has much to recommend it.

Acidosis is a frequent complication of illness, particularly if there is fever and anorexia, and, as it predisposes to the 'deoxy' state, correction of acidosis is an essential part of the management of sickle crises. The use of an induced alkalosis for treatment, however, is more debatable. In crises it seems unlikely, owing to the vascular blockage, that infused sodium bicarbonate will reach the site of the crisis in sufficient amounts to counteract the localized acidosis. Likewise the continual administration of sodium bicarbonate orally to induce an alkalosis is unlikely to succeed on two accounts. First, to maintain even a moderate alkalosis ( $\mathrm{pH} \mathrm{7.44)} \mathrm{the} \mathrm{dose} \mathrm{of} \mathrm{sodium} \mathrm{bicarbonate}$ has to be high and taken at least every four hours owing to the efficiency of renal compensation. Such administration causes unpleasant side effects which it is doubtful that most patients would accept. Secondly in a maintained alkalosis red cell metabolism is stimulated so increasing the concentration of the oxygen-linked ligand 2,3-diphosphoglycerate (2,3-DPG). In normal cells the increase in 2,3-DPG is such as to counteract exactly the effect of $\mathrm{pH}$ so that the equilibrium between the 'deoxy' and 'oxy' states remains unchanged (Bellingham, Detter, and Lenfant, 1971). It seems unlikely that the situation in sickle cells will be different in this respect and hence little chance of reducing the propensity of the cell to sickle will arise. Clinical studies on the use of induced alkalosis in both crises and as a prophylactic have failed to show any consistent benefit. It would seem therefore that the use of sodium bicarbonate should be reserved for correcting acidosis rather than for inducing alkalosis.

Avoidance of hypoxia is not usually a difficult clinical problem although the advice to give a patient with sickle cell disease regarding flying is controversial. Although a few patients do report crises following flight in commercial aircraft, where the cabin pressure is maintained equivalent to a $5000-6000 \mathrm{ft}$ altitude most patients travel by air with no sequelae. Flight in unpressurized aircraft and ascent above $10000 \mathrm{ft}$ is contraindicated. Hypoxia may occur secondary to other diseases, eg, pneumonia, and it seems prudent to increase the oxygen tension of inspired air in these cases. The few reports using hyperbaric oxygen in an attempt to abort crises are not encouraging. Hypoxia follows the use of tourniquets or arterial clamps at operation and such procedures should be avoided. It must be mentioned that orthopaedic surgeons report using exsanguinating tourniquets with no ill effects. Presumably, in these cases there are too few cells to block a capillary although they sickle under the hypoxia and on release of the tourniquet they are flushed clear in the reactive hyperaemia that ensues.

Dehydration is also likely to provoke a sickling crisis: as a result of the circulatory stagnation, both hypoxia and acidosis will occur hence maintenance of adequate hydration is essential and especially important in the anorexic and feverish patient.

\section{Anti-sickling Drugs}

Recently urea and carbamylation with cyanate or carbamyl phosphate have been suggested as antisickling agents. Urea in high concentrations in vitro stops cells sickling, possibly as a result of its ability to interfere with hydrophobic bonds which may be responsible for the polymerization. However, the concentration of urea required to inhibit significantly sickling in vitro is equivalent to a blood urea in excess of $500 \mathrm{mg} / 100 \mathrm{ml}$ (May and Huehns, 1974). Two clinical approaches have been tried using urea: (1) high dose intravenous perfusion in established crises and (2) oral urea as a prophylactic. The use of intravenous high dose urea obtained blood urea values between 200 and $400 \mathrm{mg}$ / $100 \mathrm{ml}$ and consequently required intensive medical care to maintain fluid balance in the face of the osmotic diuresis. Furthermore first reports of trials have not been encouraging. The use of regular oral urea has also been suggested but as the blood urea achieved would be only about $60 \mathrm{mg} / 100 \mathrm{ml}$ it would be unlikely to be of significant benefit. This is confirmed by the first report of a controlled trial (Lubin and Oski, 1973) although the reports of a multicentre trial are awaited.

As urea decomposes to cyanate in solution it was suggested that cyanate may have been the active agent which resulted in the initial encouraging reports of urea treatment (Cerami and Manning, 1971). Cyanate binds irreversibly to the $\mathrm{N}$-terminal valine of chains of haemoglobin (carbamylation) and is known to shift the equilibrium towards the 'oxy' conformation (Kilmartin and Rossi-Bernardi, 1969). At any given oxygen tension there is a reduced tendency to sickle with increasing fraction of the haemoglobin carbamylated. Carbamylated sickle cells however, are still capable of sickling if, fully deoxygenated. Thus carbamylation acts by shifting the equilibrium between 'oxy' and 'deoxy' states and not by directly inhibiting the intermolecular binding (May, Bellingham, Huehns, and Beaven, 1972). There are several reports showing 
that carbamylation increases red cell survival in sickle cell disease, and in a pilot clinical trial with oral sodium cyanate at a dose up to $35 \mathrm{mg} / \mathrm{kg} / \mathrm{day}$ the haemoglobin concentration increased and crises were probably reduced (Gillette, Peterson, $\mathrm{Lu}$, Manning, and Cerami, 1974). One difficulty with cyanate is that it reacts with very many proteins and its toxicity, in particular long-term toxicity, has not been fully investigated. For instance, with increasing carbamylation activities of many red cell enzymes are progressively reduced; similarly plasma proteins show different electrophoretic mobility although functional abnormalities have not been reported. Patients receiving the drug report both drowsiness and anorexia but not sufficient to withdraw the drug and one case of peripheral neuropathy has been reported. In animals receiving higher doses both possible hepatic and cerebral toxicity have been reported.

Acetylation of haemoglobin with acetyl-salicylic acid has recently been reported to have an antisickling effect by favouring the 'oxy' state (Klotz and Tam, 1973). But further studies have not substantiated this claim and thus the potential action of aspirin does not seem well founded.

\section{Summary}

Knowledge of the detailed molecular mechanisms of the sickling process has led to an understanding of factors precipitating crises and hence allowed a reasoned approach to therapy. At present amelioration of the symptoms of sickle cell disease rests primarily on the avoidance and prompt treatment of the basic precipitating factors of the sickling process-fever, hypoxia, acidosis, and dehydration. Over the years many drugs have been claimed to have anti-sickling properties but have not stood the test of time. The current partial success of cyanate stems directly from this molecular knowledge. As further details of the site of intermolecular binding involved in the sickling process become available the possibility of developing more specific anti-sickling drugs becomes clearer.

References

Bellingham, A. J., Detter, J. C., and Lenfant, C. (1971). Regulatory mechanisms of hemoglobin oxygen affinity in acidosis and alkalosis. J. clin. Invest., 50, 700-706.

Cerami, A., and Manning, J. M. (1971). Potassium cyanate as an inhibitor of the sickling erythrocytes in vitro. Proc. nat. Acad. Sci. (Wash.), 68, 1180-1183.

Finch, J. T., Perutz, M. F., Bertles, J. F., and Dobler, J. (1973). Structure of sickled erythrocytes and of sickle cell hemoglobin fibers. Proc. nat. Acad. Sci. (Wash.), 70, 718-722.

Gillette, P. N., Peterson, C. M., Lu. Y. S., Manning, J. M., and Cerami, A. (1974). Progress in the development of cyanate as a treatment for sickle cell disease. In preparation.

Huehns, E. R. (1973). Disease of haemoglobin synthesis. In Recent Advances in Medicine, edited by D. N. Baron, N. Compston, and A. M. Dawson, 16th ed., pp. 365-411. Churchill Livingstone, Edinburgh and London.

Kilmartin, J. V., and Rossi-Bernardi, L. (1969). Inhibition of CO, combination and reduction of the Bohr effect in haemoglobin chemically modified at its a-amino groups. Nature (Lond.), 222, 1243-1246

Klotz, I. M., and Tam, J. W. O. (1973). Acetylation of sickle cell hemoglobin by aspirin. Proc. nat. Acad. Sci. (Wash.), 70, 1313-1315.

Lubin, B. H., and Oski, F. A. (1973). Oral urea therapy in children with sickle cell anemia. J. Pediat., 82, 311-313.

May, A., Bellingham, A. J., Heuhns, E. R., and Beaven, G. H. (1972). Effect of cyanate on sickling. Lancet, 1, 658-661.

May, A., and Huehns, E. R. (1974. Treatment of sickle cell diseases. Trans. Roy. Soc. trop. Med., 68, 85-91.

Perutz, M. F, (1970). Stereochemistry of cooperative effects of haemoglobin. Nature (Lond.), 228, 726-739.

Perutz, M. F, (1971). Haemoglobin: genetic abnormalities. New Sci., $50,762-765$ 